\title{
Clustering of albumin excretion rate abnormalities in Caucasian patients with NIDDM
}

\author{
P.P. Faronato ${ }^{1}$, M . M aioli ${ }^{2}$, G. Tonolo ${ }^{2}$, E . B rocco ${ }^{3}$, F. Noventa ${ }^{3}$, F. Piarulli ${ }^{1}$, C. A baterusso ${ }^{1}$, F. M odena ${ }^{1}$, \\ G. de B igontina ${ }^{1}$, M. Velussi ${ }^{1}$, S. Inchiostro ${ }^{1}$, F. Santeusanio ${ }^{4}$, A . B ueti ${ }^{4}$, R. N osadini ${ }^{2,3}$ on behalf of the Italian \\ NID D M N ephropathy Study G roup
}

${ }^{1}$ Diabetic Outpatient Clinics of Feltre, Contarina, Pieve di Cadore, Monfalcone, Este and Pordenone, Italy

${ }^{2}$ Medical Clinic, University of Sassari, Sassari, Italy

${ }^{3}$ Institute of Internal Medicine and Centre of the National Research Council (CNR) for the study of Ageing, University of Padua, Padua, Italy

${ }^{4}$ Institute of Medical and Endocrinological Sciences, University of Perugia, Perugia, Italy

Summary Proteinuria and nephropathy have been found to cluster in families of non-insulin-dependent diabetic (NIDDM) Pima Indian, and in Caucasian insulin-dependent diabetic (IDDM) patients. No information is at present available for Caucasian NIDDM patients. The aim of the present study was to determine whether micro-macroalbuminuria $(\mathrm{AER}+)$ is associated with albumin excretion rate abnormalities in diabetic and non-diabetic siblings of probands with NIDDM and AER + . We identified 169 Caucasian families with one NIDDM proband (the patient with longest known NIDDM duration) (101 families with only NIDDM siblings, 33 families with both NIDDM and non-NIDDM siblings and 35 families with only non-NIDDM siblings). Of the probands 56 had AER + [Prob-NIDDM-(AER + )], 78 had AER- [Prob-NIDDM-(AER-)], 74 siblings of ProbNIDDM-(AER + ), and 113 siblings of ProbNIDDM-(AER-) also had NIDDM. Data on albuminuria and retinopathy from multiple sibling pairs when the size of the sibship was more than two was adjusted according to a weighting factor. The odds ratio for AER +, in siblings of Prob-NIDDM$(\mathrm{AER}+)$ adjusted for age, hypertension, glycated haemoglobin $A_{1 c}$ and other confounding variables was 3.94 (95\% confidence intervals: $1.93-9.01)$ as compared to siblings of Prob-NIDDM-(AER-). The 74 siblings of Prob-NIDDM-(AER + ) had higher prevalence of proliferative retinopathy than siblings of Prob-NIDDM-(AER-) (14 vs $2 \% ; p<0.01)$. We also identified 66 non-diabetic siblings of 41 NIDDM probands with AER + and 36 non-diabetic siblings of 27 NIDDM probands with AER-. Albumin excretion was two times higher, although still within the normal range, in the non-diabetic siblings of ProbNIDDM-(AER + ) than in siblings of ProbNIDDM-(AER-) $[$ median $=13.5$ (range 0.5-148) vs 6.6 (range $1-17) \mu \mathrm{g} / \mathrm{min}(\mathrm{p}<0.05)]$. In conclusion higher rates of albumin excretion aggregate in Caucasian families with NIDDM. Proliferative retinopathy is more frequently observed in families showing a clustering of AER + and NIDDM. These findings suggest that familial factors play a role in the pathogenesis of renal and retinal complications in NIDDM. [Diabetologia (1997) 40: 816-823]

Keywords Non-insulin-dependent diabetes mellitus, micro-macroalbuminuria, familial clustering, sib pair analysis, diabetic retinopathy.
Received: 6 November 1996 and in revised form: 17 February 1997

Corresponding author: Dr. P.P. Faronato, Servizio di Diabetologia ULSS 2 del Veneto, Via Bagnols sur Céze, I-32032 Feltre (BL), Italy

A bbreviations: IDDM, Insulin-dependent diabetes mellitus; NIDDM, non-insulin-dependent diabetes mellitus; AER, albumin excretion rate.
It has been suggested that genetic predisposition contributes to the development of diabetic nephropathy in insulin-dependent diabetes mellitus (IDDM). This hypothesis is based on the observation of a clustering of albuminuria and end-stage renal disease in IDDM families [1]. Although little information is available on this issue in Caucasian patients with non-insulindependent diabetes mellitus (NIDDM). Studies in Pima Indian offspring of NIDDM parents with proteinuria suggest that familial factors are associated 


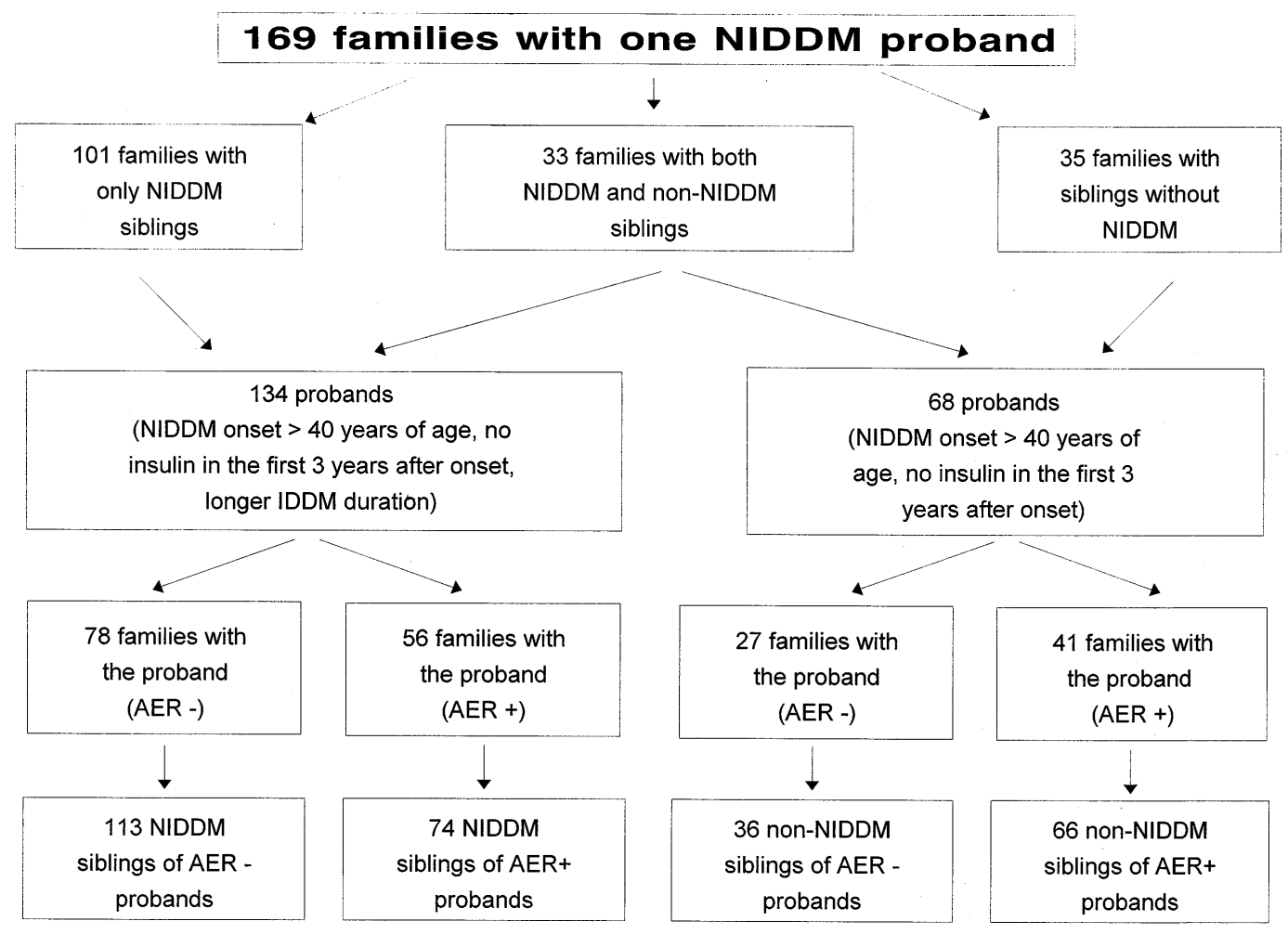

Fig. 1. Flow-chart for the identification of families in which one single NIDDM proband (the diabetic patient with the longest known duration of diabetes) was identified. The NIDDM siblings (sibling pair concordant for diabetes) and non-diabetic siblings (sibling pairs discordant for diabetes) of one single NIDDM proband in each family were recruited to complete the population of sibling pairs. Three types of families were identified: 1) only with NIDDM sibling pairs; 2) with both NIDDM and non-diabetic sibling pairs ; 3) only with discordant sibling pairs. A weighting factor was used to analyse the information contained in sibling pairs, when the size of sibship was more than two

with the development of renal disease in NIDDM [2]. This view is further supported by the finding that microalbuminuria is often found in non-diabetic subjects with a parental history of diabetes [3]. Furthermore albumin excretion rate (AER), although within the normal range, is significantly elevated in Caucasian offspring of NIDDM parents with microalbuminuria [4].

The risk of developing diabetic nephropathy appears to be associated with a genetic predisposition to arterial hypertension in IDDM $[5,6]$ and in Pima Indians [7-9]. However, among Pima Indians the increased risk of diabetic nephropathy in offspring of NIDDM parents with proteinuria and hypertension remains elevated even after being adjusted for blood pressure [2].

A well-defined constellation of renal and retinal structural abnormalities, usually described as 'retinal-renal syndrome', accompanies micro and macroalbuminuria in IDDM [10, 11].
In IDDM patients with dipstick-positive proteinuria, renal histology shows an association of mesangial expansion, increased glomerular basement membrane width and arteriolar hyalinosis in the vast majority of the subjects [10]. These renal structural lesions are accompanied by simple or proliferative diabetic retinopathy [11]. On average IDDM patients with microalbuminuria have similar but less extensive structural lesions [12], but the range of severity is wide, from values observed in normoalbuminuric patients to those observed in patients with overt proteinuria. In addition almost $50-55 \%$ of NIDDM patients have no diabetic retinopathy, despite the presence either of micro or even macroalbuminuria [13].

The aim of the present study was to determine whether there is a clustering of micro and/or macroalbuminuria (albuminuria) in Caucasian patients with NIDDM. Furthermore, the relationship between diabetic retinopathy and albuminuria was studied in the families of these patients. We also evaluated non-diabetic siblings of NIDDM probands to investigate whether familial predisposition plays a role in determining glomerular permeability to albumin, irrespective of the presence of NIDDM.

\section{Subjects and methods}

This study is a multicentre collaboration among several Diabetic Clinics in Umbria, Sardinia and the north-east region of Italy. NIDDM was diagnosed upon the following two criteria: 
1) blood glucose value above $7.8 \mathrm{mmol} / \mathrm{l}$ after overnight fast on two occasions, or higher than $10 \mathrm{mmol} / \mathrm{l}$ after a 75 -g oral glucose tolerance test [14]; 2) $\mathrm{HbA}_{1 \mathrm{c}}$ above the mean plus two standard deviations of non-diabetic control subjects $(6.5 \%)$. Patients were at least 40 years old at the onset of diabetes and not older than 75 years at the time of the study and had not required insulin therapy during the first 3 years after onset of diabetes. We identified 169 families with at least one NIDDM patient (proband). In 101 families there were only NIDDM siblings, 33 families had both NIDDM and non-NIDDM siblings. When more than one diabetic patient was found within a family only one proband (the NIDDM subject with the longest duration of the disease) was studied. In this case, all the remaining diabetic patients were used as siblings of one single proband within one family. These families comprise the population of sibling pairs concordant for NIDDM. Non-diabetic siblings of NIDDM probands were used to study the population of sibling pairs discordant for diabetes. Figure 1 shows how families of the probands and their siblings, either concordant or discordant for diabetes were identified.

There are three possible ways to define the number of sibling pairs, when the sibship is more than two: 1) inclusion of all possible sibling pairs; 2) inclusion of one proband for each individual family with all siblings; 3 ) inclusion of only one sibling pair for each family. For our study one single NIDDM proband with [P-NIDDM $(\mathrm{AER}+)]$ or without [P-NIDDM (AER-)] albuminuria was recruited for each family, whereas all siblings were taken into account to make up the sibling pair population. However, the information contained in multiple sibling pairs was reduced according to the weighting factor described by Hodge et al. [15], in agreement with the suggestion of Motro and Thomson [16]. Therefore, NIDDM siblings were divided in two groups: siblings of P-NIDDM (AER + ), [e.g. S-NIDDM $(A E R+)$ ] and siblings of P-NIDDM AER-, [e.g. S-NIDDM (AER-)]. A patient was classed as hypertensive when blood pressure was equal to or above $140 \mathrm{mmHg}$ for systolic and equal to or above $90 \mathrm{mmHg}$ for the diastolic $[17,18]$ or when they were treated by antihypertensive drugs. Blood pressure was measured on the right arm with a sphygmomanometer, after the patient had rested for $10 \mathrm{~min}$ in the supine position. Disappearance of the Korotkoff sound (phase V) was taken as the diastolic blood pressure.

Microalbuminuria was defined as a median value of AER equal to or above $30 \mu \mathrm{g} / \mathrm{min}$ in at least two out of three specimens from 24-h consecutive urine collections over a 6-month period. Macroalbuminuria was defined as a median AER above $200 \mu \mathrm{g} / \mathrm{min}$ in at least two out of three specimens from 24-h urine collections over a 6-month period. Albumin/creatinine ratio was measured in all NIDDM patients from the north-east of Italy. Misclassification of the albuminuric patients was observed in $2.3 \%$ of the probands and in $2.9 \%$ of the siblings using the cut-off points of 2.38 and $2.36 \mathrm{mg} / \mathrm{mmol}$ for men and women, respectively, rather than $30 \mu \mathrm{g} / \mathrm{min}$ of AER. Sensitivity and specificity of timed urine collections for AER have been discussed previously [19]. Patients taking antihypertensive drugs other than nifedipine and diuretics had their drug treatment withdrawn. AER was measured on three consecutive days after 12-15 days of withdrawal from other antihypertensive treatment, the patients having switched to nifedipine and diuretics. This antihypertensive treatment was mandatory from an ethical point of view. The choice of nifedipine was determined by previous, although scant, information, that AER is less influenced by nifedipine than by other antihypertensive drugs [20]. The patients were returned to their previous treatment after the assessment of AER. A threshold of $30 \mu \mathrm{g} /$ min AER was used as a cut-off point between normo and albuminuria. This more conservative approach was used instead of the traditional one usually adopted in IDDM ( $>20 \mu \mathrm{g} / \mathrm{min}$ of AER) to avoid possibly recruiting false positive microalbuminuric NIDDM patients, for whom such an abnormality is less clearly defined than in IDDM. In fact microalbuminuria, defined on the basis of the $20-200 \mu \mathrm{g} / \mathrm{min}$ cut-off point is predictive of overt nephropathy in $80 \%$ of IDDM patients [2123 ], but only in $20 \%$ of NIDDM patients [24, 25]. AER was expressed as a median of three overnight measurements in three urine specimens from three different urine collections during a 6-month period. AER was measured by radioimmunoassay techniques as described in detail elsewhere [26].

All patients followed the dietary guidelines of the Italian Diabetic Society, consisting of a low protein $\left(60 \mathrm{~g} \cdot 1.73 \mathrm{~m}^{-2}\right.$ - day $\left.^{-1}\right)$, low salt $\left(<90 \mathrm{mmol} \cdot 1.73 \mathrm{~m}^{-2} \cdot\right.$ day $\left.^{-1}\right)$, isocaloric $\left(1500 \mathrm{kcal} \cdot 1.73 \mathrm{~m}^{-2} \cdot\right.$ day $\left.^{-1}\right)$ content, which is close to the dietary habits of the normal population of the above described regions of Italy. Height and weight were measured without shoes and overcoat. The diagnosis of simple (background) and proliferative retinopathy was based on fundus oculi examination. Previous laser treatment for proliferative retinopathy was documented in the medical history of each patient in the diabetic clinics of the Universities of Padua, Sassari and Perugia. Biochemical analyses were carried out in the laboratory of the Department of Internal Medicine, University of Padua.

Glycated haemoglobin was measured by high pressure liquid chromatography [27]. Serum creatinine, serum triglycerides, total cholesterol, LDL cholesterol and HDL cholesterol were determined using standard methodology by automatic analyser [28]. Smokers were defined as those currently smoking or having smoked one or more cigarettes per day for 1 year or more. Alcohol consumption was defined by the number of drink units consumed per week (a drink unit is $285 \mathrm{ml}$ beer, $115 \mathrm{ml}$ wine or $25 \mathrm{ml}$ liquor). Coronary heart disease was diagnosed by positive response to the World Health Organisation questionnaire. A 12-lead resting ECG was recorded in the sitting position and was interpreted according to the Minnesota code to assess the diagnosis of myocardial infarction [29] by a coder who was unaware of the albuminuria status of the patient. Stroke was diagnosed by clinical evaluation and was documented by the patient having been referred to the national health system clinics for stroke treatment.

Statistical analysis. Data are expressed as mean \pm SEM or as median and range.

Comparison among the diabetic groups was assessed by one-way analysis of variance (ANOVA) with the Bonferroni pairwise multiple post-hoc test if data were normally distributed, or by Mann-Whitney test for parameters shown to be different by ANOVA.

The threshold of statistical significance was taken as $p$ less than 0.05 , all $p$ values were two-tailed. Chi-square test was used to assess distribution of hypertension, cardiovascular complications and diabetic simple and proliferative retinopathy.

AER was converted to log values because of skewed distribution. The relationship between the rates of AER in the probands and siblings was evaluated by estimating the odds ratios and confidence intervals (95\% C.I.) by multiple logistic regression, corrected for potentially confounding variables, selected for their univariate associations with AER. AER, sex, hypertension and smoking were used as dichotomous variables and age, duration of hypertension, body mass index, alcohol intake, $\mathrm{HbA}_{1 \mathrm{c}}$, duration of diabetes and the weighting factor described by Hodge et al. [15], when the sibling pair size was greater than two within one single family, as continuous variables [30]. 
Table 1. Clinical and biochemical features of NIDDM probands with [P-NIDDM(AER+)] and without albuminuria [P-NIDDM (AER-)] and of their siblings [S-NIDDM(AER+); S-NIDDM(AER-)]

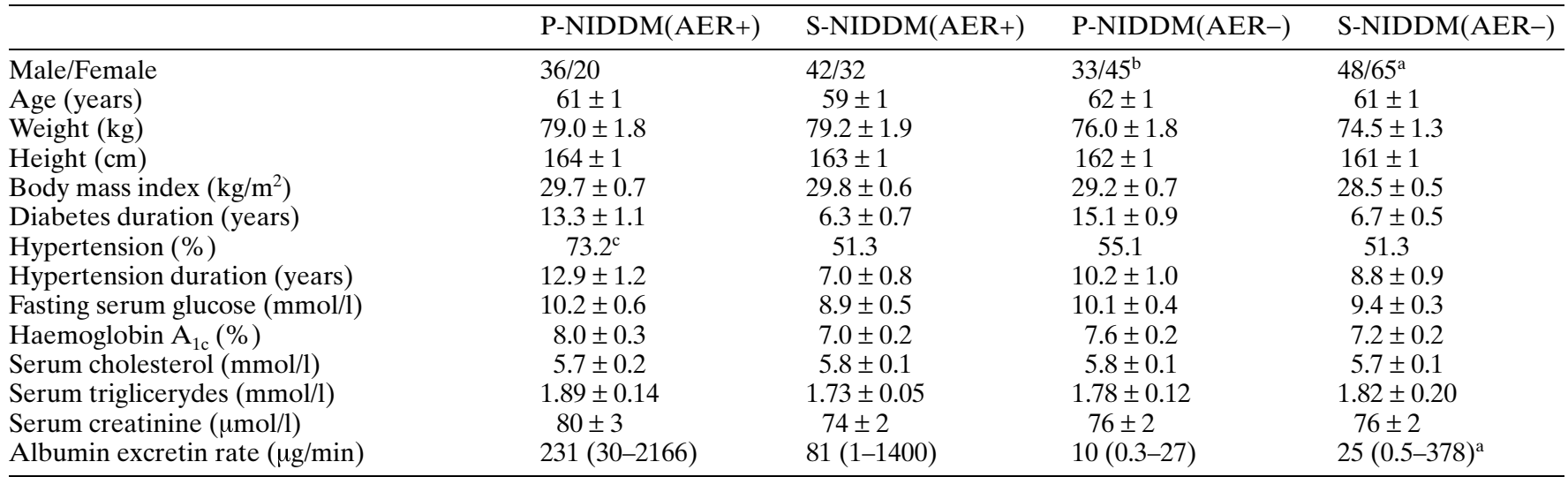

a $p<0.001$ S-NIDDM(AER+) vs S-NIDDM(AER-); ${ }^{b} p<0.01$ P-NIDDM(AER+) vs P-NIDDM(AER-); ${ }^{c} p<0.05$ P-NIDDM $(\mathrm{AER}+)$ vs all other groups

Table 2. Prevalence of arterial hypertension, macro- and microvascular complications of diabetes and treatment of diabetes in NIDDM probands with [P-NIDDM $(\mathrm{AER}+)]$ or without [P-NIDDM(AER-)] albuminuria and in their siblings [S-NIDDM $(\mathrm{AER}+) ; \mathrm{S}-\mathrm{NIDDM}(\mathrm{AER}-)]$

\begin{tabular}{lcccc}
\hline & P-NIDDM (AER+) & P-NIDDM (AER-) & S-NIDDM (AER+) & S-NIDDM (AER-) \\
\hline Myocardial infarction & 6.9 & 4.2 & 2.8 & 4.9 \\
Stroke & 6.9 & $2^{\mathrm{a}}$ & 3.9 & 1.8 \\
Treated hypertension & 67.6 & $48.2^{\mathrm{b}}$ & 40.9 & 41.2 \\
Untreated hypertension & 8.3 & 6.2 & 10.4 & 10.1 \\
Simple retinopathy & 38.2 & 30.1 & 28.1 & 14 \\
Proliferative retinopathy & 11.7 & $4^{\mathrm{a}}$ & 43.5 & 23.2 \\
Microalbuminuria & 83.3 & - & 3.6 & $2^{\mathrm{d}}$ \\
Macroalbuminuria & 16.7 & 0 & 4.5 & $13.2^{\mathrm{c}}$ \\
Elevated serum creatinine & 1.8 & 8 & 49 & $1.0^{\mathrm{d}}$ \\
Diet & 11 & 67 & 35 & 41 \\
Oral antidiabetic agents & 45 & 25 & 16 & 41 \\
Oral antidiabetic agents + insulin & 44 & & 18 \\
\hline
\end{tabular}

Values given in percent

${ }^{a} p<0.05 ;{ }^{b} p<0.01$ P-NIDDM(AER+) vs P-NIDDM(AER-); ${ }^{c} p<0.0001 ; \quad d p>0.01 ; \quad$ e $p>0.05$ S-NIDDM(AER+) vs SNIDDM (AER-)

\section{Results}

Table 1 shows the clinical and biochemical characteristics of NIDDM albuminuric [P-NIDDM(AER + )] and normoalbuminuric [P-NIDDM(AER-)] probands and their respective siblings [S-NIDD$\mathrm{M}(\mathrm{AER}+)$ and S-NIDDM(AER-)]. By definition the duration of diabetes was longer in the probands than in siblings. Male gender was more frequently found among NIDDM probands and their siblings with albuminuria [P-NIDDM(AER + ) and S-NIDD$\mathrm{M}(\mathrm{AER}+)]$ than among NIDDM probands and their siblings without albuminuria [P-NIDDM(AER-) and S-NIDDM(AER-)]. Arterial hypertension was more frequently observed in P-NIDDM(AER +) than in the other groups. No difference was observed between S-NIDDM(AER + ) and S-NIDDM(AER-) in the prevalence of hypertension. AER was significantly higher in the S-NIDDM(AER + ) than in the S-NIDDM(AER-) (81 vs $25 \mu \mathrm{g} / \mathrm{min}$, median, $p<0.001)$. No significant differences were observed with regard to other biochemical and clinical parameters. Table 2 shows the prevalence of arterial hypertension and macrovascular and microvascular complications in NIDDM probands and siblings with or without albuminuria, along with their treatment. P-NIDD$\mathrm{M}(\mathrm{AER}+)$ showed a higher prevalence of stroke, treated hypertension and proliferative retinopathy than P-NIDDM(AER-). A higher prevalence of proliferative retinopathy, corrected for known duration of diabetes and age was found in S-NIDDM(AER + ), than in S-NIDDM(AER-). No difference was observed with regard to the prevalence of simple retinopathy, although this diagnosis was based only on fundus oculi examination. No differences were found with regard to the other parameters. Albuminuria and elevated serum creatinine were almost three times more frequently observed in S-NIDDM(AER + ) than in S-NIDDM(AER-). The crude odds ratio for albuminuria was 3.94 (95\% C.I. 1.93-9.01) in 


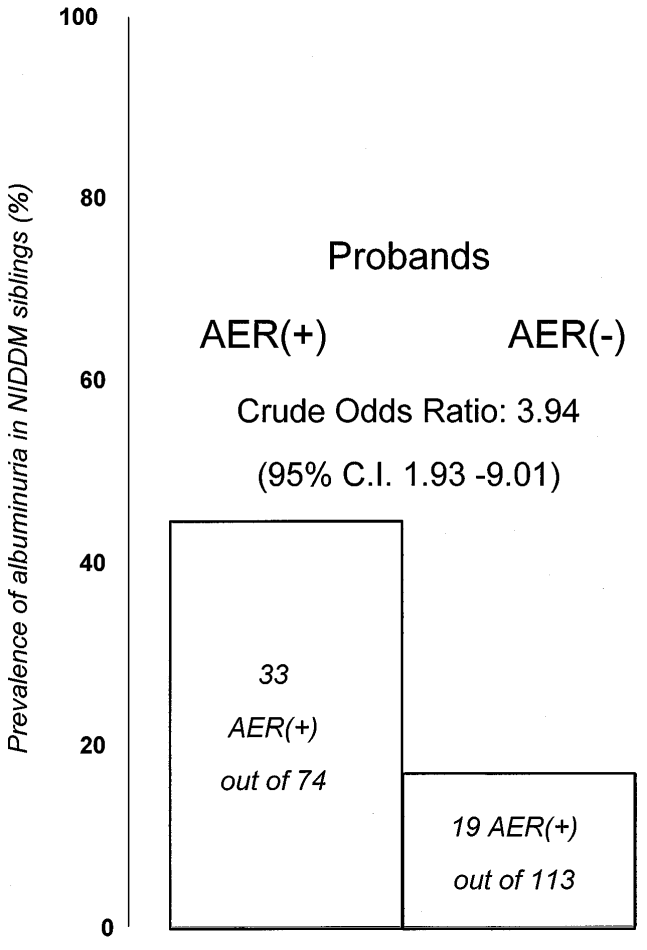

Fig. 2. Prevalence of albuminuria (AER $\geq 30 \mu \mathrm{g} / \mathrm{min}$ ) in NIDDM siblings of NIDDM probands with $[\operatorname{AER}(+)]$ in comparison with those without $[\operatorname{AER}(-)]$ albuminuria

S-NIDDM(AER + ) compared with S-NIDDM(AER-) (Fig. 2). Table 3 shows the odds ratio for albuminuria in NIDDM siblings, estimated by multiple logistic regression, adjusted for other confounding factors. The presence of albuminuria in the proband was the main variable explaining albuminuria in the sibling. Body mass index was also associated with the risk of albuminuria in S-NIDDM.

Table 4 shows the clinical and biochemical characteristics of non-diabetic siblings of the NIDDM probands with [S-N(AER + )] or without [S-N(AER-)]
Table 3. Odds ratios and $95 \%$ confidence intervals estimated by multiple logistic regression analysis and the degree of statistical significance for albuminuria in NIDDM siblings

\begin{tabular}{llll}
\hline Variable & $\begin{array}{l}\text { Odds } \\
\text { ratio }\end{array}$ & $95 \%$ C.I. & p value \\
\hline AER in probands (yes/no) & 3.94 & $1.93-9.01$ & $<0.0001$ \\
Sex (male/female) & 0.82 & $0.23-1.60$ & $\mathrm{NS}$ \\
Age (years) & 0.77 & $0.32-1.48$ & $\mathrm{NS}$ \\
Hypertension (yes/no) & 0.80 & $0.35-1.50$ & $\mathrm{NS}$ \\
Duration of hypertension (years) & 0.79 & $0.30-1.33$ & $\mathrm{NS}$ \\
${\text { Body mass index (kg/m }{ }^{2} \text { ) }}_{\text {Smoke (yes/no) }}$ & 1.21 & $1.07-1.29$ & $<0.01$ \\
Alcohol intake (g/day) $_{\text {HbA }_{1 \mathrm{c}}(\%)}$ & 0.60 & $0.81-5.40$ & $\mathrm{NS}$ \\
Duration of NIDDM (years) & 0.90 & $0.34-1.48$ & $\mathrm{NS}$ \\
\hline Albuminura & 0.82 & $0.38-1.47$ & $\mathrm{NS}$ \\
\hline
\end{tabular}

Albuminuria in the proband, sex, age, arterial hypertension, duration of hypertension, body mass index, smoke, alcohol intake, serum creatinine, $\mathrm{HbA}_{1 \mathrm{c}}$ and duration of diabetes in the siblings were the independent variables. Information contained in multiple sibling pairs was corrected using a weighting factor when sibship size was more than two [24]. The dependent variable was the presence or absence of albuminuria in the siblings

albuminuria. AER was significantly higher in the S$\mathrm{N}-\operatorname{AER}(+)$ than in the S-N-AER( $(-),(13.5 \mathrm{vs} 6.6 \mu \mathrm{g} /$ $\min , p<0.03)$. The median value for AER in the two groups of siblings was still within the normal range, although $5.2 \%$ of the overall population of non-diabetic siblings of NIDDM probands had AER in the microalbuminuric range.

\section{Discussion}

This study confirms a clustering of renal complications in Caucasian NIDDM patients, as previously reported in Pima Indians [2]. Furthermore our results indicate that microalbuminuria and increased AER, not only proteinuria, as suggested by Pettitt et al. [2] in Pima Indians, aggregate in Caucasian NIDDM

Table 4. Clinical and biochemical features of the NIDDM probands with and without albuminuria and their siblings discordant for NIDDM

\begin{tabular}{|c|c|c|c|c|}
\hline & $\begin{array}{l}\text { P-NIDDM } \\
(\mathrm{AER}+)\end{array}$ & $\begin{array}{l}\text { S-NIDDM } \\
(\mathrm{AER}+)\end{array}$ & $\begin{array}{l}\text { P-NIDDM } \\
(\mathrm{AER}-)\end{array}$ & $\begin{array}{l}\text { S-NIDDM } \\
\text { (AER-) }\end{array}$ \\
\hline Male/Female & $28 / 13$ & $26 / 40$ & $14 / 13$ & $15 / 21$ \\
\hline Age (years) & $57 \pm 1$ & $54 \pm 1$ & $58 \pm 1$ & $58 \pm 1$ \\
\hline Height $(\mathrm{cm})$ & $166 \pm 1$ & $162 \pm 1$ & $167 \pm 2$ & $164 \pm 2$ \\
\hline Body mass index $\left(\mathrm{kg} / \mathrm{m}^{2}\right)$ & $30.1 \pm 0.7$ & $27.4 \pm 0.5$ & $29.2 \pm 0.9$ & $26.4 \pm 0.8$ \\
\hline Diabetes duration (years) & $10.6 \pm 1.1$ & - & $11.7 \pm 1.2$ & - \\
\hline Fasting serum glucose $(\mathrm{mmol} / \mathrm{l})$ & $10.3 \pm 0.5$ & $5.2 \pm 0.2$ & $9.7 \pm 0.4$ & $5.3 \pm 0.2$ \\
\hline Haemoglobin $\mathrm{A}_{1 \mathrm{c}}(\%)$ & $7.5 \pm 0.3$ & $4.8 \pm 0.1$ & $8.3 \pm 0.4$ & $4.9 \pm 0.2$ \\
\hline Serum cholesterol (mmol/l) & $5.8 \pm 0.2$ & $5.8 \pm 0.1$ & $6.1 \pm 0.2$ & $5.8 \pm 0.1$ \\
\hline Serum triglicerydes $(\mathrm{mmol} / \mathrm{l})$ & $2.51 \pm 0.20$ & $1.64 \pm 0.10$ & $2.03 \pm 0.17$ & $1.53 \pm 0.14$ \\
\hline Serum creatinine $(\mu \mathrm{mol} / \mathrm{l})$ & $88 \pm 4$ & $79 \pm 2$ & $76 \pm 4$ & $76 \pm 4$ \\
\hline Albumin excretion rate $(\mu \mathrm{g} / \mathrm{min})$ & $496(37-7566)$ & $13.5(0.5-148)$ & $8.6(0.5-21)$ & $6.6(1-17)^{\mathrm{a}}$ \\
\hline
\end{tabular}

Data are expressed as mean \pm SEM; ${ }^{a} \mathrm{p}<0.03 \mathrm{~S}-\mathrm{N}(\mathrm{AER}+)$ vs $\mathrm{S}-\mathrm{N}(\mathrm{AER}-)$ 
families. Non-diabetic siblings of families with NIDDM patients with albuminuria also had increased, although still normal, AER.

The odds ratio for albuminuria after adjusting for confounding variables was 3.94 in NIDDM siblings of patients with albuminuria, compared to siblings of normoalbuminuric patients. This was mainly due to the prevalence of microalbuminuria, which was ten times higher than that of macroalbuminuria.

No differences were found for $\mathrm{HbA}_{1 \mathrm{c}}$, the prevalence of hypertension, or lipid patterns between the siblings of families with or without clustering of albuminuria.

These findings are consistent with the tenet that abnormalities in AER are determined, at least in part, independently of the metabolic challenge of diabetes, when NIDDM is present.

The odds ratio was 24.0 for siblings of probands with overt proteinuria relative to siblings with normoalbuminuria in the first report on the familial factors determining the development of diabetic nephropathy in IDDM patients [1]. The odds ratio of 3.94 observed in the present study in Caucasian NIDDM patients is closer to that found in IDDM families in Denmark [31] and in the Joslin Clinic, United States [32]. This latter report showed that the IDDM siblings of probands with advanced diabetic nephropathy had a significantly higher incidence of overt proteinuria, but that if the proband had only microalbuminuria, overt proteinuria developed in few of their siblings, similar to the siblings of the normoalbuminuric probands. The prevalence of microalbuminuria did not vary significantly among siblings with either normo, micro or macroalbuminuria [32]. However, our study demonstrates that microalbuminuria also occurs significantly more frequently in NIDDM siblings of microalbuminuric probands. It remains to be clarified whether our larger study population accounts for the different findings in comparison with the previous reports. That proliferative diabetic retinopathy clusters in Caucasian families with NIDDM and renal damage is a new finding of the present study. In fact, proliferative retinopathy was more frequently found both in NIDDM probands with albuminuria and in siblings of probands with albuminuria. On the contrary no differences were found in the prevalence of cardiovascular events and stroke between families with different clustering of renal complications. Lipid patterns and the degree of glycaemic control were likewise similar among these patients.

Microalbuminuria seems to be a more heterogeneous pathological finding in NIDDM than in IDDM. Whenever proteinuria is found in IDDM, a well-defined constellation of renal structural abnormalities, e.g. mesangial expansion, arteriolar hyalinosis and tubulo-interstitial changes, occurring in parallel can almost always be documented in kidney biopsy specimens [10]. These changes, typical of diabetic nephropathy are associated with background or proliferative diabetic retinopathy in the majority of the IDDM patients [11]. Moreover, microalbuminuria antedates overt nephropathy in $80 \%$ of microalbuminuric IDDM patients [21-23]. However, IDDM patients with microalbuminuria show similar, but less severe, lesions with the pattern of structural abnormalities scattered over a wide spectrum ranging from values overlapping those observed in normoalbuminuric patients to those found in patients with overt nephropathy [33]. More recently the EURODIAB IDDM Complications study [34] showed that diabetic retinopathy is not so frequently observed as previously thought in microalbuminuric IDDM patients and is strictly associated with increased circulating levels of von Willebrand factor, a marker of endothelial dysfunction. On the contrary, more than half the NIDDM patients have no diabetic retinopathy, despite having micro- and even macroalbuminuria. In addition microalbuminuria is antecedent to overt nephropathy in only $20 \%$ of NIDDM patients [24, 25].

Using light microscopy we have recently reported structural heterogeneity in renal biopsy specimens from microalbuminuric NIDDM patients [12]. In particular $29 \%$ of these patients have normal or nearnormal renal structure, $29 \%$ have changes typical of diabetic nephropathy and $42 \%$ have important tubulo-interstitial fibrosis and/or arteriolar hyalinosis and/or glomerular sclerosis with changes of relatively trivial glomerular abnormalities. Proliferative retinopathy was only found in patients with changes typical of diabetic nephropathy.

The findings of the present study support the view that albuminuria to some extent reflects pathogenic mechanisms in common with retinal complications in a subgroup of NIDDM patients as in the ocular-renal syndrome in IDDM. However, it is likely that albuminuria is of more complex significance than the mere expression of renal damage in the overall population of NIDDM patients, since the clustering of disorders of AER occurs in a larger cohort of patients independent of diabetic retinopathy. This latter finding is also true if we correct the rate of albuminuria and retinal changes for age and known duration of diabetes. However, it should be pointed out that the link between the development of ocular-renal complications in NIDDM and its duration has not been clearly described.

Our data do not show a familial aggregation of arterial hypertension with albuminuria. Nelson et al. [8] recently reported that hypertension in both parents is a risk factor for proteinuria in diabetic Pima Indian offspring. These authors suggested that the underlying mechanism for this relationship could be the existence of factors that confer susceptibility to both hypertension and diabetic renal disease. However, the same report did not show a significant association between parental hypertension and blood 
pressure in offspring after controlling for parental diabetes, in agreement with a previous study in Pima Indian children [34]. Further studies are needed to clarify whether arterial hypertension clusters in Caucasian NIDDM families as it does in Pima Indians contemporaneously with renal damage. However arterial hypertension is frequently found in as many as $80 \%$ of NIDDM patients even when renal function is normal, and albuminuria determines only a slight further increase in the prevalence of hypertension [11]. Thus, it can be postulated that diabetes and hypertension are closely linked in the overall NIDDM population, whereas renal complications develop only in a much smaller cohort of these patients.

In conclusion the present study demonstrates that albuminuria clusters in families with NIDDM and that AER is higher in siblings of NIDDM probands with albuminuria, even when they are not diabetic.

It was also found that diabetic proliferative retinopathy is associated with albuminuria. An increased permeability to albumin, albeit within normal range, was observed in the siblings of NIDDM probands with albuminuria, even in the absence of diabetes.

These findings suggest that susceptibility to altered AER is familial in Caucasian NIDDM, at least partially independent of diabetes and the predisposition to hypertension. Proliferative retinopathy also tends to cluster in a subgroup of families with NIDDM patients with AER abnormalities. It remains to be clarified whether these complications are inherited or occur in later adulthood, due to acquired familial factors.

A cknowledgements. We acknowledge the skilled technical help of Ms. S. I. Barzon and L. Occhionero. This study was supported by a grant (9300428 and 9400464.P.F. I. 40) from the National Research Council and CNR $60 \%$ National Research Council, 1995-1996, University of Sassari.

\section{The I talian NID D M N ephropathy Study G roup}

University of Genoa; G. Deferrari; Diabetes and Endocrinology Department-Mestre: F. Virgili, F. Frigato, A Carraro; Diabetic Clinic Contarina: M. Sambataro; Diabetic Clinic Camposampiero: M. R. Cipollina, E. Schirru; Diabetic Clinic Este: B. Muollo; Department of Endocrinology and Metabolic Diseases-University of Ancona: F. Mantero, G. Giacchetti; Diabetic Clinic, Medical Clinic - University of Sassari: M. Ciccarese, P. Fresu, G. Realdi; Clinical Medical Institute - University of Ferrara: A. Solini; Internal Medicine Institute University of Padua: R. Trevisan, M. Bruseghin, A. Saller, P. Fioretto, G. Crepaldi.

\section{References}

1. Seaquist ER, Goetz FC, Rich S, Barbosa J (1989) Familial clustering of diabetic kidney disease. N Engl J Med 320: 1161-1165

2. Pettitt D, Saad M, Bennett PH, Nelson RG, Knowler WC (1990) Familial predisposition to renal disease in two generations of Pima Indians with type 2 (non-insulin-dependent) diabetes mellitus. Diabetologia 33: 438-443

3. Haffner S, Gonzales C, Valdez R et al. (1993) Is microalbuminuria part of the prediabetic state? The Mexico City Diabetes Study. Diabetologia 36: 1002-1006

4. Gruden G, Cavallo-Perin P, Olivetti C, Repetti E, Sivieri R, Bruno A, Pagano G (1995) Albumin excretion rate levels in non-diabetic offspring of NIDDM patients with and without nephropathy. Diabetologia 38: 1218-1222

5. Viberti GC, Keen H, Wiseman M (1987) Raised arterial pressure in parents of proteinuric insulin dependent diabetics. BMJ 295: 515-517

6. Krolewski A, Canessa M, Warram J, Laffei L, Christlier R, Knowler WC, Rand L (1988) Predisposition to hypertension and susceptibility to renal disease in insulin-dependent diabetes mellitus. N Engl J Med 318: 140-145

7. Kunzelman CL, Knowler WC, Pettitt DJ, Bennet PH et al. (1989) Incidence of nephropathy in type 2 diabetes mellitus in the Pima Indians. Kidney Int 35: 681-687

8. Nelson R, Pettitt DJ, Baird HR (1993) Prediabetic blood pressure predicts urinary albumin excretion after the onset of type 2 (non-insulin-dependent) diabetes mellitus in Pima Indians. Diabetologia 33: 998-1001

9. Nelson R, Pettitt D, de Courten M et al. (1996) Parental hypertension and proteinuria in Pima Indians with NIDDM. Diabetologia 39: 433-438

10. Mauer M (1994) Structural-functional correlations of diabetic nephropathy. Kidney Int 45: 612-622

11. Parving HH, Hommel E, Mathiesen E et al. (1988) Prevalence of microalbuminuria, arterial hypertension, retinopathy and neuropathy in patients with insulin dependent diabetes. BMJ 296: 156-160

12. Fioretto P, Mauer M, Brocco E et al. (1996) Patterns of renal injury in type 2 NIDDM patients with microalbuminuria. Diabetologia 39: 1569-1576

13. Gall A-M, Rossing P, Skott P et al. (1991) Prevalence of micro- and macroalbuminuria, arterial hypertension, retinopathy and large vessel disease in European type 2 (non-insulin dependent) diabetic patients. Diabetologia 34: 655-661

14. National Diabetes Group (1979) Classification and diagnosis of diabetes mellitus and other categories of glucose intolerance. Diabetes 26: 1039-1058

15. Hodge SE (1984) The information contained in multiple sibling pairs. Genet Epidemiol 1: 109-122

16. Motro U, Thomson G (1985) The affected sib method. I. Statistical features of the affected sib pair method. Genetics 110: 525-538

17. Joint National Committee on Detection, Evaluation and Treatment of Hight Blood Pressure (1993) The fifth report on the joint national committee on detection, evaluation and treatment of high blood pressure (JNC-V). Arch Intern Med 153: 154-183

18. American Diabetes Association (1993) Treatment of hypertension in diabetes. Diabetes Care 16: 1394-1401

19. Mangili R, Deferrari G, Di Mario U et al. (1992) Prevalence of hypertension and microalbuminuria in adult type I diabetic patients without renal failure in Italy. Validation of screening techniques to detect microalbuminuria. The Italian Microalbuminuria Study Group. Acta Diabetol 29: 156-166

20. Kasiske BL, Kalil R, Ma J et al. (1993) Effect of antihypertensive therapy on the kidney in patients with diabetes: a meta-regression analysis. Am Intern Med 118: 129-138

21. Viberti GC, Hill RD, Jarret RJ, Argyropoulos A, Mahmud U, Keen H (1982) Microalbuminuria as a predictor of clinical nephropathy in insulin-dependent diabetes mellitus. Lancet I:1430-1432 
22. Parving HH, Oxenboll R, Svensen PAA, Christiansen JS, Andersen AR (1982) Early detection of patients at risk of developing nephropathy: a longitudinal study of urinary albumin excretion. Acta Endocrinol Copenh 100: 550-552

23. Mogensen CE, Christensen CK (1986) Predicting diabetic nephropathy in insulin-dependent diabetic patients. $\mathrm{N}$ Engl J Med 331: 89-93

24. Mogensen CE (1984) Microalbuminuria predicts clinical proteinuria and early mortality in maturity-onset diabetes. N Engl J Med 310: 356-360

25. Mogensen CE (1987) Microalbuminuria as a predictor of clinical diabetic nephropathy. Kidney Int 31: 673-689

26. Keen H, Chlouverakis C (1963) An immunoassay method for urinary albumin at low concentrations. Lancet ii:913914

27. Dunn PJ, Cole RA, Soeldner JS (1979) Further development and automation of a high pressure liquid chromatographic method for the determination of $\mathrm{HbA}_{1 \mathrm{c}}$. Metabolism 28: 777-779

28. Care RN, Feldbruegger D, Westgard J (1974) Evaluation of the adaptation of the glucose peroxide 3-methyl-2 benzothiazolinone hydrazone-N-N dimethyline procedure to the technicon "SMA" 12/60 and comparison with other automated methods of glucose determination. Clin Chem 20: 595-602
29. Rose GA, Blackburn H (1968) Cardiovascular survey methods. World Health Organisation Geneva pp 137-142

30. Hosmer DW, Lemeshow S (1980) Applied logistic regression. John Wiley and Sons, New York

31. Borch-Johnsen K, Norgaard K, Hommel E et al. (1992) Is diabetic nephropathy an inherited complication? Kidney Int 41: 719-722

32. Quinn M, Angelico MC, Warram JH, Krolewski AS (1996) Familial factors determine the development of diabetic nephropathy in patients with IDDM. Diabetologia 39: 940-945

33. Fioretto P, Steffes MW, Mauer M (1994) Glomerular structure in non proteinuric IDDM patients with various levels of albuminuria. Diabetes 43: 1358-1364

34. Karamanos B, Fuller JH, Stevens LK and the Eurodiab Study Group (1996) Short duration of IDDM with microangiopathy vs long duration without risk: determinants and protective factors. Diabetologia 39 [Suppl]:A26 (Abstract)

35. Charles MA, Pettitt DJ, Hanson RL et al. (1994) Familial and metabolic factors related to blood pressure in Pima Indian children. Am J Epidemiol 140: 123-131 